\title{
Health Care Resource Utilization and Costs Among Newly Diagnosed and Oral Anticoagulant-Naive Nonvalvular Atrial Fibrillation Patients Treated with Dabigatran or Warfarin in the United States
}

\author{
Rahul Jain, PhD; An-Chen Fu, MS; Jonathan Lim, MSc; Cheng Wang, MD, PhD; \\ Jessica Elder, PhD; Stephen D. Sander, PharmD; and Hiangkiat Tan, BPharm, MS
}

\begin{abstract}
BACKGROUND: Warfarin has a long history of use to reduce the risk of stroke in patients with atrial fibrillation (AF), but it requires frequent laboratory monitoring to maintain international normalized ratio levels in the therapeutic range. Dabigatran, a novel oral anticoagulant (OAC), has demonstrated efficacy in reducing the risk of stroke and systemic embolism and does not require laboratory monitoring.
\end{abstract}

OBJECTIVE: To compare health care resource utilization (HCRU) and costs of OAC-naive patients newly diagnosed with nonvalvular atrial fibrillation (NVAF), using dabigatran or warfarin.

METHODS: This retrospective observational study used data from medical and pharmacy claims extracted from the HealthCore Integrated Research Database representing commercial and Medicare Advantage members. Adults aged $>18$ years with a medical diagnosis claim of NVAF were identified between 0ctober 1, 2010, and December 31, 2011. The date of first observed OAC prescription claim was the index date. Patients were followed for up to 12 months after the index date. Patients were assigned to the dabigatran or warfarin treatment groups based on their first OAC prescription fills. To reduce potential for selection bias, the cohorts were matched on baseline characteristics using propensity score matching. HCRU was measured and compared between groups on a per-patient-per-month (PPPM) basis for all-cause HCRU, as well as stroke, myocardial infarction, and bleed-specific HCRU. Pharmacy, medical, and total costs were also compared and adjusted to 2012 U.S. dollars. Generalized linear models were conducted to compare all-cause health care costs between cohorts.

RESULTS: After propensity score matching, 1,648 patients were included in the analysis (824 each in the dabigatran and warfarin treatment groups). In the post-index period, patients in the dabigatran group had significantly fewer all-cause PPPM physician office visits (mean [SD] $1.29[ \pm 0.95]$ vs. $2.02[ \pm 1.53], P<0.001$ ) and outpatient visits (mean [SD] $2.17[ \pm 2.90]$ vs. $3.52[ \pm 3.32], P<0.001)$ compared with those in the warfarin group. There were no between-group differences in outcomes for the number of stroke, myocardial infarction, or bleeding-related office visits. All-cause medical costs for the dabigatran cohort were lower than the warfarin cohort; however, the difference did not reach statistical significance $(\$ 2,696[S D \pm \$ 6,699]$ vs. $\$ 2,893[ \pm \$ 6,819], P=0.179)$. All-cause pharmacy costs were higher in the dabigatran group versus the warfarin group (\$455 [ $\pm \$ 429]$ vs. $\$ 328[ \pm \$ 517], P<0.001)$. The dabigatran cohort also had significantly higher stroke-related (\$32 [ \pm \$11] vs. \$20 [ \pm \$55], $P=0.006)$ and nonstroke-related pharmacy costs $(\$ 423[ \pm \$ 422]$ vs. $\$ 308$ $[ \pm \$ 515], P<0.001)$. Despite higher pharmacy costs for the dabigatran cohort, both treatment groups had statistically similar all-cause total costs $(\$ 3,151[ \pm \$ 6,744]$ vs. $\$ 3,221[ \pm \$ 6,869], P=0.701)$.

CONCLUSIONS: This real-world study showed that among patients newly diagnosed with NVAF who were OAC naive, dabigatran use was associated with significantly less HCRU in terms of physician and outpatient visits but higher pharmaceutical costs in up to 12 months of follow-up. Similar to other real-world studies, this research supports the finding that higher pharmacy costs for dabigatran users was offset by lower medical costs, making total health care costs comparable between dabigatran and warfarin.

J Manag Care Spec Pharm. 2018;24(1):73-82

Copyright $\odot$ 2018, Academy of Managed Care Pharmacy. All rights reserved.

\section{What is already known about this subject}

Atrial fibrillation (AF) is an independent risk factor for stroke, and anticoagulation therapy is critical to reduce the risk of stroke among patients with AF.

Warfarin has a long history of use to reduce the risk of stroke in patients with $\mathrm{AF}$ but requires frequent laboratory monitoring, whereas dabigatran, a novel oral anticoagulant (OAC), has demonstrated efficacy in reducing the risk of stroke and does not require laboratory monitoring.

Previous studies have shown lower emergency department and outpatient visits and comparable total health care costs for dabigatran users among newly diagnosed and newly treated nonvalvular (NV) AF patients.

\section{What this study adds}

In addition to including driver-related (stroke, bleed, and MI) health care resource utilization (HCRU) and cost analyses, this study strengthens the findings from previous studies.

This study found, as expected, higher pharmaceutical costs for the branded dabigatran users; however, the use of dabigatran was associated with less HCRU in terms of physician and outpatient visits. Components of lower medical costs offset higher pharmacy costs (in up to 12 months follow-up period) for dabigatran users, making the total cost comparable between dabigatran and warfarin users.

A trial fibrillation (AF), the most common type of arrhythmia, affects as many as $1 \%$ of the U.S. population. ${ }^{1}$ In the United States, more than $95 \%$ of all AF cases are classified as nonvalvular (NV), ${ }^{2}$ which is AF in the absence of mitral stenosis or valvular prostheses. ${ }^{3} \mathrm{AF}$ is an independent risk factor for stroke, ${ }^{4}$ which more than quadruples a person's 
stroke risk and accounts for $15 \%$ of all strokes in the United States, of which $30 \%$ are among people aged 80 to 89 years. ${ }^{4,5}$ It is expected that more than 12 million people in the United States will have AF by 2030.1,6

The economic outcomes of stroke events can be devastating. The estimated direct cost of stroke in the United States was $\$ 28.3$ billion in 2010 and is expected to increase to $\$ 95.6$ billion by 2030, with indirect costs (including loss of productivity) estimated to grow from $\$ 25.6$ billion in 2010 to $\$ 44.4$ billion by $2030 .{ }^{4}$ In a study using real-world data, Naccarelli et al. (2015) showed that NVAF patients who had a stroke or major bleeding event had substantially higher health care-related costs. ${ }^{7}$ The authors analyzed administrative claims from U.S. commercial and Medicare health plans and found that patients who had strokes incurred $\$ 4,669$ higher health care costs (all-cause unadjusted per patient per month [PPPM]) than patients with NVAF who did not have strokes or major bleeding. ${ }^{7}$

To reduce the risk of stroke in patients with AF, anticoagulation therapy is critical. ${ }^{8,9}$ Oral anticoagulants (OACs) are easy to administer and are recommended for patients with AF to reduce the long-term risk of stroke. ${ }^{10}$ The $\mathrm{CHADS}_{2}$ score, a composite measure of the presence of congestive heart failure, hypertension, age (75 years or older), diabetes mellitus, and previous stroke or transient ischemic attack, is a validated predictor of stroke risk. ${ }^{11}$ Antithrombotic guidelines issued by the American College of Chest Physicians recommend OACs for patients with an intermediate risk of stroke $\mathrm{CHADS}_{2}$ score of 1 ) and OACs rather than aspirin or a combination of aspirin and clopidogrel for patients with a high risk of stroke $\left(\mathrm{CHADS}_{2}\right.$ score of 2 or higher). ${ }^{12}$

Warfarin has a long history of reducing stroke risk in patients with $\mathrm{AF}^{10}$ However, managing patients on warfarin can be challenging because of the need to achieve and maintain the therapeutic level of international normalized ratio (INR) between 2.0 and 3.0. Values outside this range can lead to overor undercoagulation, ${ }^{13,14}$ which in turn may lead to thrombosis or bleeding. Despite the importance of maintaining warfarin within a therapeutic range, it has been shown that nearly $50 \%$ of AF patients who have a stroke have inadequate anticoagulation (INR $<1.5$ ) values, and 30\% of patients are not taking warfarin at the time of their strokes. ${ }^{13}$ Moreover, studies have shown that patients maintain a target INR level only approximately $50 \%$ of the time, ${ }^{15}$ and up to one third of AF patients newly initiated on warfarin discontinue therapy within the first year of treatment. ${ }^{16,17}$ Since INR monitoring is required at least monthly, and sometimes as often as twice weekly, ${ }^{18}$ such coordinated care has the potential to substantially increase cost. A 2010 systematic review of 29 studies found that the cost of a single INR monitoring test ranged from $\$ 6.19$ to $\$ 145.70$ in 2006 U.S. dollars. ${ }^{19}$
Compared with warfarin, newer OACs have a valued role in reducing the risk of stroke and improving health outcomes for patients with $\mathrm{AF}$, since they do not require frequent coagulation monitoring and associated dose adjustments, which can lead to savings in health care costs. Dabigatran, the first OAC to become available in more than 50 years, was approved by the U.S. Food and Drug Administration in 2010 for the prevention of stroke in patients with NVAF. ${ }^{20}$ The dabigatran clinical trial Randomized Evaluation of Long-Term Anticoagulation Therapy (RE-LY) demonstrated that the agent was associated with significantly lower rates of stroke and systemic embolism, as well as fewer intracranial hemorrhages, compared with warfarin. ${ }^{21}$ As a patented medication, dabigatran has higher pharmacy costs than warfarin, which is a generic product. Dabigatran has been shown to have a higher persistence rate than warfarin, ${ }^{22}$ which also may add to pharmacy costs. Offsetting these pharmacy costs, however, is the reduced need for frequent INR monitoring, which is not required for those receiving dabigatran, as well as lower rates of associated complications such as stroke, systemic embolism, and intracranial hemorrhages. ${ }^{21}$

Recent retrospective studies have shown that among newly diagnosed NVAF patients, dabigatran users, in comparison with warfarin users, had lesser length of stay for AF-related hospitalizations, along with associated costs; however, they had similar 30-day readmission rates. ${ }^{23}$ Other studies have evaluated allcause health care resource utilization (HCRU) and costs and found that dabigatran use was associated with lower resource utilization across all settings and that costs were similar despite higher pharmacy-related costs. ${ }^{24,25} \mathrm{~A}$ recent study also evaluated HCRU by specific drivers. ${ }^{25}$ This study found that stroke-related hospitalizations and physician office visits and bleed-related emergency department (ED) visits were lower for the dabigatran cohort compared with the warfarin cohort.

Given the better efficacy of branded dabigatran and no need for expensive INR testing, the purpose of our study was to explore the overall and driver-related effect of using dabigatran over warfarin on HCRU and costs for patients with NVAF, using real-world data.

\section{Methods}

\section{Data Source}

In this retrospective observational study, medical and pharmacy claims and eligibility information were extracted from the HealthCore Integrated Research Database (HIRD), which is one of the largest datasets of commercially insured U.S. residents, containing claims from 14 geographically dispersed health plans representing 45 million lives. HIRD is comparable with U.S. Census data, with the HIRD population being slightly younger, since all members are commercially insured.

All claims data used were deidentified and accessed with protocols compliant with the Health Insurance Portability and Accountability Act of 1996 regulations. No waiver of informed consent was required from an institutional review board. 


\section{Study Design}

Medical and pharmacy claims between October 1, 2009, and December 31, 2012, were used in the analysis. This period was defined as the study period. The index date for each patient was defined as the date of the first OAC prescription between October 1, 2010, and December 31, 2011; the first observed OAC prescription was identified as the index OAC. The 12 months before the index date was considered the pre-index period.

To increase the likelihood of association between outcomes and the index OAC, patients were followed for up to 12 months after the index date and were censored on the discontinuation date of the index OAC, switch to a different anticoagulant from the index OAC, disenrollment from the health plan, or end of the observation period, whichever occurred first. Since the patients had variable follow-up, HCRU and costs during the follow-up period were calculated on a PPPM basis. Patients included in the analysis had Medicare Advantage or were commercially insured.

\section{Study Population}

Adults who were aged 18 years or older with newly diagnosed NVAF and who were OAC naive were identified. Patients were considered newly diagnosed if the claim for the first observed AF diagnosis was on the index date or within 3 months before the index date. ${ }^{22}$ Patients were considered OAC naive if they did not have a pharmacy claim for an OAC (warfarin and dabigatran) during the pre-index period (12 months before the index date).

Patients were included in the analysis if they were continuously enrolled in a health plan during the 12-month pre-index period, although no minimum eligibility was required during the follow-up period. During the pre-index period, all patients were required to have at least 1 inpatient hospitalization, 2 physician office visits, or 2 ED visits or 1 office and 1 ED visit with a diagnosis of AF on distinct service dates. To ensure that patients were truly treated with index OACs, they were required to have least 2 fills of the index OAC during the follow-up period, including a fill on the index date. To define NVAF, patients were excluded from the analysis if they had a diagnosis of hyperthyroidism (International Classification of Diseases, Ninth Revision, Clinical Modification [ICD-9-CM] diagnosis code 242.x), ${ }^{26}$ a medical claim for valvular heart disease in the pre-index period (Appendix A, available in online article), or a claim within 3 months of the first observed diagnosis of $\mathrm{AF}$ for cardiac surgery (ICD-9-CM procedure codes 00.5x, 35.xx, 36.xx, or 37.xx); pericarditis (ICD-9-CM diagnosis codes 391.x, 393, 420.x, 423.2, 0.36.41, 074.21, 093.81, or 098.83); myocarditis (ICD-9-CM diagnosis codes 391.2, 422.xx, 074.23, 398.0, 429.0, 032.43, 093.82, or 130.3); or pulmonary embolism (ICD-9-CM diagnosis code 415.1x).
Patients were assigned to either the dabigatran (Generic Product Identifier [GPI] code starting with 8337030) or warfarin (GPI code starting with 83200030) treatment group based on their first OAC prescription fill. Discontinuation of the index OAC medication was defined as a treatment gap of more than 30 days from the end of the calculated days supplied.

\section{Pre-index Characteristics}

Patient demographic characteristics, including age, gender, geographic region, and type of health plan, were captured at baseline. In addition, the provider specialty of the OAC prescribing physician was also recorded.

At the pre-index assessment, comorbidities were evaluated using the Deyo-Charlson Comorbidity Index (DCI) and the Elixhauser Comorbidity Index (ECI). ${ }^{27,28}$ Other specific comorbidities assessed during the pre-index period included cancer, rheumatoid arthritis, coronary artery disease, acute myocardial infarction (MI), cardiomyopathy, ischemic stroke, transient ischemic attack, heart failure, atrial flutter, hypertension, peripheral artery disease, liver disease, renal disease, chronic obstructive pulmonary disease/emphysema, diabetes, peptic ulcer/gastroesophageal reflux disease, venous thromboembolism, hyperlipidemia, thrombocytopenia, chronic anemia, coagulopathy, and bleeding at any position. Stroke risk was assessed for the pre-index period using the $\mathrm{CHADS}_{2}$ and $\mathrm{CHADS}_{2}$-VASc (congestive heart failure/left ventricular dysfunction, hypertension, age 75 years or older [doubled], diabetes, stroke [doubled], vascular disease, age 65-74 years, sex category [female]), stroke risk scores, ${ }^{29,30}$ and bleeding risk was assessed using the HEMORR ${ }_{2}$ HAGES score (hepatic/renal disease, ethanol abuse, malignancy history, older age [greater than 75 years], reduced platelet count or function, rebleeding risk [history of past bleeding], hypertension [uncontrolled], anemia, genetic factors, excessive fall risk, and stroke history). Pre-index use of enoxaparin, dalteparin fondaparinux, beta blockers, calcium channel blockers, diuretics, other antihypertensives, antihyperlipidemics, corticosteroids, antidiabetics, antiarrhythmics, antiplatelets, ketoconazole, and nonsteroidal anti-inflammatory drugs was also assessed. ${ }^{31}$ The time to index OAC was defined as the period between the first AF diagnosis observed in the pre-index period and the index date.

\section{Propensity Score Matching}

To reduce potential selection bias, the study cohorts were matched on baseline characteristics using the propensity score matching method. The nearest neighbor method of propensity score matching within a caliper of 0.02 was used to select the matched samples. Propensity score regression controlled for patient demographics (e.g., age and gender); health plan type; geographic region; quarter of index date; pre-index medical and pharmacy expenditure; specialty of prescriber of the 


\section{TABLE 1 Attrition for Commercially Insured and Medicare Advantage Patients}

Total patients in HIRD during patient identification period

Patients with at least 1 inpatient or 2 physician office visits or emergency department visits or a combination of the 2 on distinct service dates with diagnosis of AF (ICD-9-CM diagnosis code 427.31, at any position) during study period
$\mathrm{N}=13,582,416$

$n=148,986$

\begin{tabular}{|c|c|c|c|c|}
\hline \multirow{2}{*}{$\begin{array}{l}\text { Patients with at least } 1 \text { pharmacy claim of dabigatran or warfarin during patient } \\
\text { identification period }\end{array}$} & \multicolumn{2}{|c|}{$\begin{array}{l}\text { Dabigatran Cohort } \\
\mathrm{n}\end{array}$} & \multicolumn{2}{|c|}{$\begin{array}{l}\text { Warfarin Cohort } \\
\text { n }\end{array}$} \\
\hline & \multicolumn{2}{|c|}{8,641} & \multicolumn{2}{|c|}{41,311} \\
\hline & $\mathrm{n}$ & Remaining \% & n & Remaining \% \\
\hline Patients continuously enrolled in a health plan for a minimum of 12 months before index date & 7,080 & 81.9 & 31,431 & 76.1 \\
\hline Patients aged 18 years or older on index date & 7,079 & 100.0 & 31,427 & 100.0 \\
\hline Patients without diagnosis of hyperthyroidism within 12 months before index date & 6,932 & 97.9 & 30,794 & 98.0 \\
\hline $\begin{array}{l}\text { Patients without cardiac surgery, pericarditis, myocarditis, or pulmonary embolism within } \\
3 \text { months before first AF diagnosis }\end{array}$ & 6,751 & 97.4 & 29,619 & 96.2 \\
\hline Patients without valvular heart disease within 12 months before index date & 6,644 & 98.4 & 28,615 & 96.6 \\
\hline Patients left after exclusion of those who were previously treated with warfarin & 6,308 & 94.9 & 27,655 & 96.6 \\
\hline Patients left after exclusion of those with multiple OACs on index date & 6,286 & 99.7 & 27,655 & 100.0 \\
\hline Patients with at least 2 fills of index OAC during post-index period (including index fill) & 4,646 & 73.9 & 22,001 & 79.6 \\
\hline Patients newly diagnosed with NVAF and OAC treatment naive & 1,197 & 25.8 & 2,501 & 11.4 \\
\hline Patients previously diagnosed with NVAF and OAC treatment naive & 959 & 80.1 & 2,220 & 88.8 \\
\hline
\end{tabular}

AF = atrial fibrillation; HIRD = HealthCore Integrated Research Database; ICD-9CM=International Classification of Diseases, Ninth Revision, Clinical Modification; NVAF= nonvalvular atrial fibrillation; OAC=oral anticoagulant.

index OAC; pre-index (DCI) score ${ }^{32}$; stroke risk scores (i.e., $\mathrm{CHADS}_{2}$ ); bleeding risk scores (i.e., HEMORR ${ }_{2} \mathrm{HAGES}$ ); preindex comorbidities; pre-index medications; and time from the first observed AF diagnosis to index OAC treatment.

To test the robustness of the results and compare against the study sample, a sensitivity analysis was performed on OAC-naive patients previously diagnosed with NVAF. Patients were considered as previously diagnosed if the claim for first observed AF diagnosis appeared between 12 and 3 months before the index date.

\section{Outcomes of Interest}

HCRU was assessed for the entire follow-up period using information from medical and pharmacy claims. Because of variable follow-up, HCRU was measured on a PPPM basis and was calculated by dividing the total number of ED, inpatient hospital, physician office, and outpatient visits by the days of follow-up and multiplying the result by 30 .

Pharmacy, medical, and total costs (medical plus pharmacy costs) were assessed. All costs were adjusted to 2012 U.S. dollars using the medical component of the Consumer Price Index.

HCRU and costs were evaluated in terms of type of service (e.g., inpatient hospitalization, ED visit, or physician office visit) for all-cause, as well as stroke-related, conditions (i.e., ischemic stroke ICD-9-CM diagnosis codes 433.x1, 434.x1, and 436 at any position ${ }^{33}$ ); MI-related conditions (ICD-9-CM diagnosis codes 410.xx at any position); and bleeding-related conditions (e.g., gastrointestinal bleeding or intracranial bleeding ${ }^{34}$; Appendix B, available in online article). The AF-related pharmacy costs included pharmacy costs for cordarone, moricizine hydrochloride, propafenone and flecainide, dronedarone, betapace, dofetilide, disopyramide and Quinidine.

\section{Statistical Analysis}

All study variables were analyzed descriptively. Means ( \pm standard deviation [SD]) and medians were reported for continuous variables, and frequencies (\%) were reported for categorical variables. For the paired cohort comparison, the McNemar test was used for nominal variables; the Wilcoxon signed rank test was used for ordinal variables or discrete non-normal continuous variables; and paired t-tests were used for interval variables.

The multivariable analyses were conducted for the PPPM total cost (i.e., medical plus pharmacy costs). Because of the non-normal distribution and skewed nature of cost data, statistical comparisons were conducted using a generalized linear model (GLM) approach. Because a negligible number of patients ( 3 of 1,648 patients) had zero cost, a 2-part cost model was not used. The appropriate family of the distribution was chosen using the modified Park test GLMs with gamma distribution, and log link function was used to estimate the difference in costs between the dabigatran and warfarin treatment groups. The appropriateness of using log link for the chosen family of distribution was tested using the modified Park test and modified Hosmer-Lemeshow goodness-of-fit test. 
Health Care Resource Utilization and Costs Among Newly Diagnosed and Oral Anticoagulant-Naive Nonvalvular Atrial Fibrillation Patients Treated with Dabigatran or Warfarin in the United States

TABLE 2 Baseline Demographic Characteristics of Patients Newly Diagnosed with NVAF in OAC-Naive Dabigatran and Warfarin Cohorts Before and After Propensity Score Matching

\begin{tabular}{|c|c|c|c|c|c|c|c|c|c|c|}
\hline \multirow[b]{2}{*}{ Variables } & \multicolumn{5}{|c|}{ Before PSM } & \multicolumn{5}{|c|}{ After PSM } \\
\hline & \multicolumn{2}{|c|}{$\begin{array}{c}\text { Dabigatran Cohort } \\
(\mathrm{n}=1,197)\end{array}$} & \multicolumn{2}{|c|}{$\begin{array}{l}\text { Warfarin Cohort } \\
\qquad(\mathrm{n}=2,501)\end{array}$} & $\begin{array}{c}P \\
\text { Value }^{\mathrm{a}}\end{array}$ & \multicolumn{2}{|c|}{$\begin{array}{c}\text { Dabigatran Cohort } \\
(\mathrm{n}=824)\end{array}$} & \multicolumn{2}{|c|}{$\begin{array}{l}\text { Warfarin Cohort } \\
\qquad(\mathrm{n}=824)\end{array}$} & \multirow[t]{2}{*}{$\begin{array}{c}P \\
\text { Value }^{\mathrm{b}}\end{array}$} \\
\hline \multicolumn{10}{|l|}{ Gender, n (\%) } & \\
\hline Male & 866 & $(72)$ & 1,476 & (59) & & 551 & $(67)$ & 556 & $(67)$ & \\
\hline Female & 331 & (28) & 1,025 & (41) & $<0.001$ & 273 & (33) & 268 & (33) & 0.791 \\
\hline \multicolumn{11}{|l|}{ Age, years } \\
\hline Age, mean (SD) & 63 & $( \pm 11.2)$ & 69 & $( \pm 12.4)$ & $<0.001$ & 64 & $( \pm 11.6)$ & 64 & $( \pm 11.9)$ & 0.759 \\
\hline \multicolumn{11}{|l|}{ Region of residence, $\mathrm{n}(\%)$} \\
\hline South & 399 & $(33)$ & 539 & $(22)$ & & 236 & $(29)$ & 235 & $(29)$ & \\
\hline Midwest & 356 & (30) & 1,044 & $(42)$ & & 281 & (34) & 277 & (34) & \\
\hline Northeast & 247 & $(21)$ & 605 & $(24)$ & & 196 & $(24)$ & 195 & $(24)$ & \\
\hline West & 195 & (16) & 313 & (13) & $<0.001$ & 111 & (13) & 117 & (14) & 0.981 \\
\hline \multicolumn{11}{|l|}{ Health plan type } \\
\hline $\mathrm{PPO}$ & 759 & $(63)$ & 1,266 & $(51)$ & & 493 & $(60)$ & 512 & $(62)$ & \\
\hline $\mathrm{HMO}$ & 280 & (23) & 977 & (39) & & 226 & $(27)$ & 208 & $(25)$ & \\
\hline Medicare Advantage only & 185 & $(15)$ & 1,094 & $(44)$ & $<0.001$ & 185 & $(22)$ & 176 & $(21)$ & 0.537 \\
\hline CDHP & 100 & (8) & 114 & $(5) S$ & 0.079 & 61 & $(7)$ & 56 & $(7)$ & 0.617 \\
\hline FFS & 58 & $(5)$ & 144 & $(6)$ & & 44 & $(5)$ & 48 & (6) & \\
\hline \multicolumn{11}{|l|}{ Selected comorbidities, n (\%) } \\
\hline Hypertension & 797 & $(67)$ & 1,938 & $(77)$ & $<0.002$ & 584 & $(71)$ & 574 & $(70)$ & 0.599 \\
\hline Hyperlipidemia & 563 & $(47)$ & 1,253 & $(50)$ & 0.081 & 387 & $(47)$ & 396 & $(48)$ & 0.651 \\
\hline Coronary artery disease & 315 & (26) & 949 & (38) & $<0.001$ & 249 & $(30)$ & 259 & $(31)$ & 0.583 \\
\hline Deyo-Charlson Comorbidity Index score, mean (SD) & 1.52 & $( \pm 1.81)$ & 2.63 & $( \pm 2.39)$ & $<0.001$ & 1.85 & $( \pm 1.96)$ & 1.91 & $( \pm 1.94)$ & 0.760 \\
\hline Elixhauser Comorbidity Index score, mean (SD) & 4.13 & $( \pm 2.03)$ & 5.43 & $( \pm 2.56)$ & $<0.001$ & 4.51 & $( \pm 2.12)$ & 4.54 & $( \pm 2.15)$ & 0.666 \\
\hline $\mathrm{CHADS}_{2}$ stroke risk score, mean (SD) & 1.40 & $( \pm 1.14)$ & 2.10 & $( \pm 1.33)$ & $<0.001$ & 1.62 & $( \pm 1.18)$ & 1.60 & $( \pm 1.18)$ & 0.815 \\
\hline HEMORR2HAGES bleeding risk score, mean (SD) & 1.37 & $( \pm 1.23)$ & 2.33 & $( \pm 1.65)$ & $<0.001$ & 1.60 & $( \pm 1.31)$ & 1.63 & $( \pm 1.33)$ & 0.450 \\
\hline \multicolumn{11}{|l|}{ Concomitant medications (by class), n (\%) } \\
\hline Beta blockers & 606 & $(51)$ & 1,241 & $(50)$ & 0.567 & 404 & $(49)$ & 410 & $(50)$ & 0.759 \\
\hline Calcium channel blockers & 343 & $(29)$ & 703 & $(28)$ & 0.730 & 243 & $(29)$ & 244 & $(30)$ & 0.957 \\
\hline Diuretics & 330 & (28) & 863 & $(35)$ & $<0.001$ & 259 & $(31)$ & 250 & $(30)$ & 0.635 \\
\hline Other antihypertensives & 639 & (53) & 1,345 & $(54)$ & 0.822 & 446 & $(54)$ & 443 & $(54)$ & 0.885 \\
\hline \multicolumn{11}{|l|}{ Index OAC prescribing provider specialty, n (\%) } \\
\hline Cardiology & 536 & $(45)$ & 628 & $(25)$ & & 309 & (38) & 314 & (38) & \\
\hline Primary care physician $^{\mathrm{c}}$ & 203 & (17) & 936 & $(37)$ & & 194 & $(24)$ & 183 & $(22)$ & \\
\hline Other/unknown & 458 & (38) & 937 & (37) & $<0.001$ & 321 & (39) & 327 & $(40)$ & 0.809 \\
\hline Time to index OAC, ${ }^{\mathrm{d}}$ mean days (SD) & 19.50 & $( \pm 23.11)$ & 19.65 & $( \pm 22.72)$ & 0.001 & 18.70 & $( \pm 22.58)$ & 18.73 & $( \pm 22.64)$ & 0.975 \\
\hline Pre-index medical costs, mean (SD) & 20,018 & $( \pm 30,749)$ & 36,992 & $( \pm 65,226)$ & $<0.001$ & 23,360 & $( \pm 34,990)$ & 24,724 & $( \pm 31,184)$ & 0.082 \\
\hline Pre-index pharmacy costs, mean (SD) & 2,947 & $( \pm 5,787)$ & 2,759 & $( \pm 6,926)$ & 0.079 & 2,845 & $( \pm 4,800)$ & 2,979 & $( \pm 4,947)$ & 0.991 \\
\hline \multicolumn{11}{|c|}{$\begin{array}{l}\text { aT-test or Wilcoxon signed rank test was used for continuous variables, and chi-square test was used for categorical variables. } \\
\text { bMcNemar test was used for nominal variables; Wilcoxon test was used for ordinal variables or discrete/non-normal continuous variables; and paired t-test was used for } \\
\text { continuous variables. } \\
\text { Includes internal medicine and family/general practice. } \\
\text { dTime from the first observed atrial fibrillation diagnosis to the index OAC refill, in days. } \\
\text { CDHP= consumer-directed health plan; FFS = fee for service; HMO=health maintenance organization; NVAF=nonvalvular atrial fibrillation; OAC=oral anticoagulant; } \\
\text { PPO= preferred provider organization; PSM= propensity score matching; SD = standard deviation. }\end{array}$} \\
\hline
\end{tabular}

The multivariable model was adjusted for patient demographics, geographic region, health plan types, ECI score, $\mathrm{CHADS}_{2}$ score, HEMORR ${ }_{2}$ HAGES score, and index OAC provider specialty. All data analyses were conducted using SAS version 9.1 (SAS Institute, Cary, NC) or Stata version 11.2 (StataCorp, College Station, TX).

\section{Results}

\section{Baseline Characteristics}

A total of 26,647 patients met the study inclusion criteria (4,646 in the dabigatran treatment group and 22,001 in the warfarin treatment group), of which 3,698 patients (1,197 in the dabigatran treatment group and 2,501 in the warfarin 


\section{Health Care Resource Utilization and Costs Among Newly Diagnosed and Oral Anticoagulant-Naive Nonvalvular Atrial Fibrillation Patients Treated with Dabigatran or Warfarin in the United States}

treatment group) were classified as newly diagnosed NVAF and OAC naive (Table 1). Compared with those in the warfarin group, patients in the dabigatran group were younger; a higher percentage were men; and a lower percentage had Medicare Advantage as their health plan. Patients in the dabigatran group also had lower baseline stroke risk and bleeding risk scores than those in the warfarin group (Table 2).

After using propensity score matching, $68.8 \%$ of the dabigatran patients were matched 1-to-1 with warfarin patients, and 1,648 patients remained (824 each in the dabigatran and warfarin treatment groups; mean age $64[\mathrm{SD} \pm 12]$ years; and $67 \%$ men). The mean post-index eligibility of was $322(S D \pm 89)$ days for the dabigatran cohort and $318( \pm 92)$ days for the warfarin cohort. Within these matched cohorts, 572 patients (69.4\%) in the dabigatran and 548 patients (66.5\%) in the warfarin cohorts $(P<0.001)$ were censored due to discontinuation or switching of the index medication. The matched cohorts were comparable across all studied characteristics, including baseline comorbidity scores and stroke and bleeding risk scores (Table 2).

The most common comorbidities were hypertension $(71 \%$ dabigatran vs. $70 \%$ warfarin, $P=0.599$ ), hyperlipidemia (47\% dabigatran vs. $48 \%$ warfarin, $P=0.651$ ), and coronary artery disease (30\% dabigatran vs. $31 \%$ warfarin, $P=0.583$ ). Antihypertensives, including angiotensin-converting enzyme inhibitors, angiotensin II receptor antagonists, and direct renin inhibitors, were the most frequently used medication class in both treatment groups.

HCRU and costs in the post-index period were evaluated and compared within the matched cohorts.

\section{Post-index Health Care Resource Utilization}

Inpatient hospitalizations were numerically lower for the dabigatran cohort but were statistically similar to the warfarin cohort during the post-index period (Table 3). Patients in the dabigatran group had significantly fewer all-cause PPPM physician office visits (mean 1.29 [SD \pm 0.95$]$ vs. 2.02 [SD \pm 1.53 ], $P<0.001)$ and fewer all-cause PPPM outpatient visits in the post-index period (mean 2.17 [SD \pm 2.90$]$ vs. 3.52 [SD \pm 3.32$]$, $P<0.001)$ than those in the warfarin group. There were no between-group differences in the number of stroke-, MI-, or bleeding-related office visits.

\section{Post-index Health Care Costs}

The all-cause medical costs for the dabigatran cohort were lower than the warfarin cohort; however, the difference did not reach statistical significance $(\$ 2,696$ [SD $\pm \$ 6,699$ ] PPPM dabigatran vs. $\$ 2,893[\mathrm{SD} \pm \$ 6,819]$ PPPM warfarin, $P=0.179$; Table 4). All-cause PPPM pharmacy costs were higher in the dabigatran group compared with the warfarin group $(\$ 455$ [SD $\pm \$ 429$ ] vs. $\$ 328$ [SD $\pm \$ 517], P<0.001)$, respectively. The dabigatran cohort also had significantly higher PPPM AF-related pharmacy costs $(\$ 32[S D \pm \$ 71]$ vs. $\$ 20[S D \pm \$ 55], P=0.006)$ and

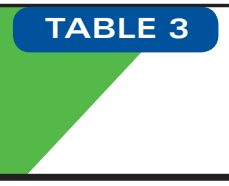

Health Care Resource Utilization of Patients Newly Diagnosed with NVAF in OAC-Naive Dabigatran and Warfarin Cohorts After Propensity Score Matching

\begin{tabular}{|c|c|c|c|}
\hline Resource Utilization ${ }^{a}$ & $\begin{array}{c}\text { Dabigatran } \\
\text { Cohort } \\
(\mathrm{n}=824) \\
\text { Mean (SD) }\end{array}$ & $\begin{array}{c}\text { Warfarin } \\
\text { Cohort } \\
(\mathrm{n}=824) \\
\text { Mean (SD) }\end{array}$ & $\begin{array}{c}P \\
\text { Value }^{b}\end{array}$ \\
\hline \multicolumn{4}{|l|}{ Post-index hospitalizations } \\
\hline All-cause & $0.08( \pm 0.20)$ & $0.09( \pm 0.29)$ & 0.866 \\
\hline Stroke-related & $0.00( \pm 0.02)$ & $0.01( \pm 0.08)$ & 0.271 \\
\hline Myocardial infarction-related & $0.00( \pm 0.06)$ & $0.01( \pm 0.09)$ & 0.433 \\
\hline Bleed-related & $0.00( \pm 0.03)$ & $0.00( \pm 0.04)$ & 0.455 \\
\hline \multicolumn{4}{|c|}{ Post-index emergency department visits } \\
\hline All-cause & $0.04( \pm 0.13)$ & $0.04( \pm 0.14)$ & 0.760 \\
\hline Stroke-related & $0.00( \pm 0.01)$ & $0.00( \pm 0.02)$ & 0.999 \\
\hline Myocardial infarction-related & $0.00( \pm 0.01)$ & $0.00( \pm 0.01)$ & 0.564 \\
\hline Bleed-related & $0.00( \pm 0.02)$ & $0.00( \pm 0.02)$ & 0.110 \\
\hline \multicolumn{4}{|c|}{ Post-index physician office visits } \\
\hline All-cause & $1.29( \pm 0.95)$ & $2.02( \pm 1.53)$ & $<0.001$ \\
\hline Stroke-related & $0.02( \pm 0.17)$ & $0.02( \pm 0.11)$ & 0.496 \\
\hline Myocardial infarction-related & $0.00( \pm 0.02)$ & $0.00( \pm 0.06)$ & 0.083 \\
\hline Bleed-related & $0.00( \pm 0.00)$ & $0.00( \pm 0.00)$ & 0.564 \\
\hline \multicolumn{4}{|l|}{ Post-index outpatient visits } \\
\hline All-cause & $2.17( \pm 2.90)$ & $3.52( \pm 3.32)$ & $<0.001$ \\
\hline Stroke-related & $0.06( \pm 0.87)$ & $0.04( \pm 0.33)$ & 0.085 \\
\hline Myocardial infarction-related & $0.01( \pm 0.15)$ & $0.01 \quad( \pm 0.14)$ & 0.345 \\
\hline Bleed-related & $0.00( \pm 0.05)$ & $0.01( \pm 0.08)$ & 0.123 \\
\hline
\end{tabular}

a Health care resource utilization (number of visits) was calculated as PPPM. ${ }^{b}$ Wilcoxon signed rank test was used.

NVAF = nonvalvular atrial fibrillation; $O A C=$ oral anticoagulant $P P P M=$ per patient per month; $S D=$ standard deviation

non-AF-related pharmacy costs $(\$ 423[\mathrm{SD} \pm \$ 422]$ vs. $\$ 308$ $[S D \pm \$ 515], P<0.001)$, respectively. Both treatment groups had statistically similar all-cause total costs, although the point estimate was lower for the dabigatran cohort $(\$ 3,151$ $[\mathrm{SD} \pm \$ 6,744]$ vs. $\$ 3,221[\mathrm{SD} \pm \$ 6,869], P=0.701)$.

Costs for inpatient hospitalizations, ED visits, and outpatient visits were similar between the 2 treatment groups for all-cause stroke-, MI-, and bleeding-related costs in the post-index period (Table 4). All-cause PPPM physician office visit costs were significantly lower in the dabigatran group (\$197 [SD $\pm \$ 545])$ compared with the warfarin group (\$232 [SD $\pm \$ 351], P<0.001$ ); however, stroke-, MI-, and bleedingrelated physician office visit costs were similar between the 2 groups. The dabigatran cohort had lower outpatient visit costs than the warfarin cohort; however, the difference did not reach statistical significance ( $\$ 946[S D \pm \$ 2,015]$ vs. $\$ 1,067$ $[S D \pm \$ 2,350], P=0.621)$.

\section{Multivariable Analysis}

After adjusting for baseline health care costs, demographics, geographic region, insurance type, ECI score, $\mathrm{CHADS}_{2}$ score, 
TABLE 4 Health Care Costs for Patients Newly Diagnosed with NVAF in OAC-Naive Dabigatran and Warfarin Cohorts After Propensity Score Matching

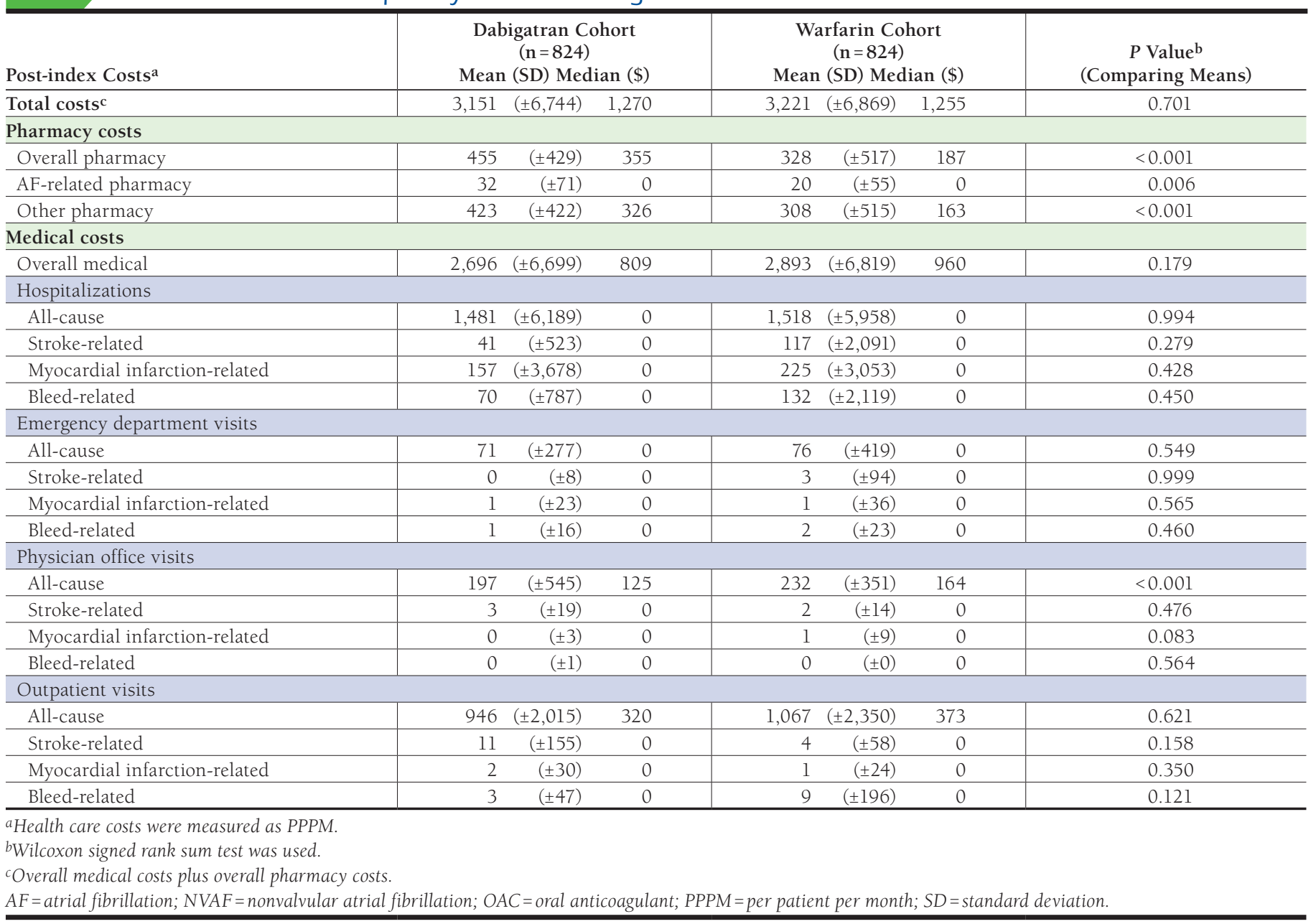

HEMORR ${ }_{2}$ HAGES score, and OAC prescriber specialty, no difference was observed in total all-cause PPPM costs between the 2 cohorts $(0.35 \%, 95 \%$ confidence interval $[\mathrm{CI}]=-17.24 \%$ $19.98 \%, P=0.97$; with warfarin as the reference group). Adjusted mean total costs were predicted from the GLM model for patients in the dabigatran and warfarin cohorts, while keeping all other variables constant at their mean values. The predicted adjusted total costs of patients using dabigatran $(\$ 2,949)$ and patients using warfarin $(\$ 2,959)$ were comparable.

\section{Sensitivity Analysis}

To test the robustness of the results, the analysis was repeated on patients previously diagnosed with NVAF who were OAC naive, where patients were considered as previously diagnosed if the claim for first observed AF diagnosis appeared between 12 and 3 months before the index date.
Of the 26,647 patients who met the inclusion criteria, 3,179 patients (959 in the dabigatran treatment group and 2,220 in the warfarin treatment group) were classified as previously diagnosed with NVAF and OAC naive. Of these patients, 1,380 patients were 1-to-1 matched (690 patients each in the dabigatran and warfarin treatment groups). The matched cohorts were comparable across all studied characteristics

Similar to the main analysis, the post-index inpatient hospitalizations (all-cause mean 0.08 [SD \pm 0.19 ] vs. 0.10 [SD \pm 0.25$]$, $P=0.417$ ) and $E D$ visits (all-cause mean 0.04 [SD \pm 0.16$]$ vs. $0.05[S D \pm 0.16], P=0.635$ ) were numerically lower but statistically similar for the dabigatran cohort compared with the warfarin cohort. Among these previously diagnosed OACnaive patients, those assigned to the dabigatran cohort had significantly fewer all-cause physician office visits (mean 1.12 [SD \pm 1.00$]$ vs. 1.55 [SD \pm 1.30$], P<0.001)$ and outpatient visits 
(mean $1.76[\mathrm{SD} \pm 1.93]$ vs. $2.80[\mathrm{SD} \pm 2.75], P<0.001$ ), when compared with patients assigned to the warfarin cohort. This result was consistent with the main analysis.

The all-cause PPPM medical costs for the dabigatran cohort $(\$ 3,562[\mathrm{SD} \pm \$ 7,717])$ was numerically lower but statistically similar compared with the warfarin cohort $(\$ 3,841$ $[\mathrm{SD} \pm \$ 8,812], P=0.890)$. As expected, the all-cause pharmacy costs were higher in the dabigatran group compared with the warfarin group. Both treatment groups had statistically similar all-cause PPPM total costs $(\$ 3,562[\mathrm{SD} \pm \$ 7,717]$ dabigatran vs. $\$ 3,841$ [SD $\pm \$ 8,812]$ warfarin, $P=0.894$ ). In addition to the similarity of costs related to inpatient hospitalizations, ED visits, and outpatient visits across the 2 cohorts-as observed in the main analysis - the costs related to office visits were also similar between the 2 cohorts in this analysis. In the main analysis, the costs related to office visits were significantly lower for the dabigatran cohort.

\section{Discussion}

Warfarin has long been used to reduce the risk of stroke in patients with AF but requires regular monitoring to maintain appropriate therapeutic levels. ${ }^{10}$ The emergence of pharmaceutical alternatives to warfarin, such as apixaban, edoxaban, dabigatran, and rivaroxaban, has given prescribers and patients additional anticoagulation choices, offering more convenient administration while eliminating the need for laboratory testing. ${ }^{18,35}$ In addition, clinical trials have demonstrated that dabigatran is associated with significantly lower rates of stroke and systemic embolism, as well as fewer intracranial hemorrhages, compared with warfarin, ${ }^{21}$ and a recent real-world study has shown that the cost of stroke-related hospitalizations is high and varies by stroke type. ${ }^{36}$ The average cost of ischemic stroke was $\$ 18,963$ $(\mathrm{SD} \pm \$ 21,454)$; hemorrhagic stroke was $\$ 32,035(\mathrm{SD} \pm \$ 32,046)$; and other strokes were found to be $\$ 19,248$ (SD \pm \$21,703).

Using administrative claims data, our study focused on newly diagnosed and newly treated patients and did so to eliminate any potential bias due to length of treatment and treatment history, including restarts and switching of treatments. Our results indicated that dabigatran use was associated with a significantly lower number of physician office visits than warfarin use and significantly lower all-cause physician office visit costs in up to 12 months after treatment initiation. Patients taking dabigatran do not require coagulation monitoring, ${ }^{35}$ as opposed to patients taking warfarin, who must maintain a therapeutic level near the target INR of 2.5 (range 2.0-3.0) to avoid the problems of over- or undercoagulation. ${ }^{13,14}$ INR monitoring for patients taking warfarin is recommended at least once monthly and, at times, as often as twice weekly. ${ }^{18}$ The difference in the need for laboratory testing may explain the lower number of all-cause physician office visits and allcause outpatient visits for the dabigatran group.
Our study found that patients in the dabigatran group had higher pharmacy costs. A previous observational study of patients newly diagnosed with NVAF showed higher persistence rates among patients taking dabigatran than patients taking warfarin at 6 months (72\% dabigatran vs. 53\% warfarin, $P<0.001)$ and 1 year $(63 \%$ dabigatran vs. $39 \%$ warfarin, $P<0.001$ ), which in part may explain the higher pharmacy costs. ${ }^{22}$ Also, patented dabigatran has a higher unit price relative to generic warfarin, which explains why pharmacy costs were higher in the dabigatran group compared with the warfarin group.

This study also found that in up to 12 months of follow-up, physician office costs were significantly lower for the dabigatran cohort, which was related to the lower number of physician office visits. Inpatient, ED, and outpatient visit costs were lower for the dabigatran users, although the difference did not reach statistical significance. The total medical costs-defined as the sum of inpatient, ED, and physician office and outpatient visit costs-was statistically comparable between the 2 treatment groups; however, the point estimate was lower for the dabigatran cohort.

The total cost, which is the sum of pharmacy costs and total medical cost, was similar between the 2 treatment groups. Pharmacy costs accounted for approximately 14\% of the total cost in the dabigatran cohort and approximately 10\% of the total cost for the warfarin cohort. Despite higher pharmacy costs, total health care costs were similar in both cohorts in part because of lower medical costs driven by lower HCRU in the dabigatran cohort.

Previously published clinical trials and real-world studies demonstrate that, compared with warfarin, dabigatran is associated with significantly lower rates of stroke and systemic embolism, as well as intracranial hemorrhages ${ }^{21}$; has higher persistence rates ${ }^{22}$; fewer physician office and outpatient visits; and, despite higher pharmacy costs, comparable total costs.

Among several safe and effective OACs, formulary decision makers must also weigh the economic effect of each OAC. In addition to having a positive effect on health outcomes and HCRU for patients with NVAF, ${ }^{21}$ the use of dabigatran may also help improve the efficient use of medical resources. The results of this study support the findings of previous real-world studies reporting that medical costs offset higher pharmacy costs, which result in comparable costs between warfarin and dabigatran in the first 12 months after treatment initiation. ${ }^{24}$

\section{Limitations}

This study has some limitations to consider. Similar to other database studies, this study is subject to possible coding errors of omission and commission, incomplete claims, unreliable clinical coding, and unobservable factors that may have influenced outcomes. All patients were commercially insured 


\section{Health Care Resource Utilization and Costs Among Newly Diagnosed and Oral Anticoagulant-Naive Nonvalvular Atrial Fibrillation Patients Treated with Dabigatran or Warfarin in the United States}

or had Medicare Advantage insurance, so the results may not be generalizable to patients outside of the United States or to those with other types of health insurance.

Only direct costs were evaluated, so total costs may have been underestimated. Also, medication use was identified from the first observed pharmacy claim during the patient identification period. Any specific medications dispensed during hospitalization were not identified to assign patients to the treatment groups or to assign index date. Furthermore, the patients were followed for a maximum of 12 months, so the results may not be generalized to a longer period of time.

For direct health care costs, other costs outside of administrative data, such as over-the-counter drug use (e.g., aspirin use), were not captured and could have been underestimated.

This study focused only on patients that had at least 2 index OAC prescriptions to ensure that patients were actually treated with an index OAC; consequently, the findings might not be applicable to those patients who were exposed once only and never refilled. Also, patients were followed and censored when they discontinued or switched their index OACs. This approach might have excluded some follow-up time; however, it strengthened the association and attributability between the outcomes and index OACs by assessing HCRU and costs only for the period when patients were being treated with the index OACs.

\section{Conclusions}

This real-world study evaluated the effect of dabigatran use compared with warfarin on HCRU and costs and showed that, among patients newly diagnosed with NVAF and who were OAC naive, dabigatran use was associated with fewer physician office visits than warfarin. During the 12-month follow-up period, this study found that dabigatran use was associated with significantly higher pharmacy costs; however, the total health care costs were comparable between dabigatran and warfarin. Further studies are warranted to evaluate and quantify how clinical event rates drive differences in HCRU and cost between dabigatran and warfarin users.

\section{Authors}

RAHUL JAIN, PhD; AN-CHEN FU, MS; and HIANGKIAT TAN, BPharm, MS, HealthCore, Wilmington, Delaware. JONATHAN LIM, MSc; CHENG WANG, MD, PhD; JESSICA ELDER, PhD; and STEPHEN D. SANDER, PharmD, Boehringer Ingelheim Pharmaceuticals, Ridgefield, Connecticut.

AUTHOR CORRESPONDENCE: Rahul Jain, PhD, Research Manager, HealthCore, 300 Brickstone Sq., 8th Fl., Andover, MA 01810. E-mail: Rahul.jain@gmail.com.

\section{DISCLOSURES}

This work was supported by Boehringer Ingelheim Pharmaceuticals, which is the manufacturer of dabigatran, one of the products included in the analysis of this work. The authors were responsible for all content and editorial decisions. Jain and Tan are employed by HealthCore, a research consultancy which was funded by Boehringer Ingelheim Pharmaceuticals for work on this study. Fu was employed by HealthCore at the time of this study. Lim, Wang, Elder, and Sander are employees of Boehringer Ingelheim Pharmaceuticals.

Study concept and design were contributed by Wang, Sander, and Tan, along with Fu and Jain. Fu, Tan, and Jain collected the data, and data interpretation was performed by Lim, Wang, and Sander, along with Jain, Tan, and Fu. The manuscript was written by Jain, Elder, Tan, and Wang, along with Lim and Fu, and revised by Jain, Wang, Elder, and Tan.

Some of the results of this study were presented at Quality of Care and Outcomes Research in Cardiovascular Disease and Stroke (QCOR) 2014 Scientific Sessions on June 2-4, 2014, in Baltimore, Maryland.

\section{REFERENCES}

1. Colilla S, Crow A, Petkun W, Singer DE, Simon T, Liu X. Estimates of current and future incidence and prevalence of atrial fibrillation in the US adult population. Am J Cardiol. 2013;112(8):1142-47.

2. Go AS, Hylek EM, Phillips KA, et al. Prevalence of diagnosed atrial fibrillation in adults: national implications for rhythm management and stroke prevention: the Anticoagulation and Risk Factors in Atrial Fibrillation (ATRIA) Study. JAMA. 2001;285(18):2370-75.

3. Stettin GD. Treatment of nonvalvular atrial fibrillation. West J Med. 1995;162(4):331-39. Available at: http://www.ncbi.nlm.nih.gov/pmc/articles/ PMC1022771/pdf/westjmed00056-0039.pdf. Accessed November 17, 2017.

4. Wolf PA, Singer DE. Preventing stroke in atrial fibrillation. Am Fam Physician. 1997;56(9):2242-50.

5. Wolf PA, Abbott RD, Kannel WB. Atrial fibrillation as an independent risk factor for stroke: the Framingham Study. Stroke. 1991;22(8):983-88. Available at: http://stroke.ahajournals.org/content/22/8/983.long. Accessed November 17, 2017

6. Romero JR, Morris J, Pikula A. Stroke prevention: modifying risk factors. Ther Adv Cardiovasc Dis. 2008;2(4):287-303. Available at: http://www.ncbi. nlm.nih.gov/pmc/articles/PMC2729177/pdf/nihms-115556.pdf. Accessed November 17, 2017

7. Naccarelli G, Stokes M, DeLeon A, et al. Healthcare costs following stroke and major bleeding events in nonvalvular atrial fibrillation patients. Circulation Cardiovasc Qual Outcomes. 2015;8(Suppl 2):301 [Abstract]. Available at: http://circoutcomes.ahajournals.org/content/8/Suppl_2/A301. Accessed November 17, 2017.

8. Hyers TM, Aggnelli G, Hull RD, et al. Antithrombotic therapy for venous thromboembolic disease. Chest. 2001;119(1 Suppl):176S-93S.

9. Albers GW, Sherman DG, Gress DR, Paulseth JE, Peterson P. Stroke prevention in nonvalvular atrial fibrillation: a review of prospective randomized trials. Ann Neurol. 1991;30(4):511-18.

10. Amin A. Oral anticoagulation to reduce risk of stroke in patients with atrial fibrillation: current and future therapies. Clin Interv Aging. 2013;8:75-84. Available at: http://www.ncbi.nlm.nih.gov/pmc/articles/PMC3556861/pdf/ cia-8-075.pdf. Accessed November 17, 2017.

11. Rietbrock S, Heeley E, Plumb J, van Staa T. Chronic atrial fibrillation: incidence, prevalence, and prediction of stroke using the Congestive heart failure, Hypertension, Age > 75, Diabetes mellitus, and prior Stroke or transient ischemic attack (CHADS2) risk stratification scheme. Am Heart J. 2008;156(1):57-64. 


\section{Health Care Resource Utilization and Costs Among Newly Diagnosed and Oral Anticoagulant-Naive Nonvalvular Atrial Fibrillation Patients Treated with Dabigatran or Warfarin in the United States}

12. You JJ, Singer DE, Howard PA, et al. Antithrombotic therapy for atrial fibrillation: antithrombotic therapy and prevention of thrombosis, 9th ed: American College of Chest Physicians Evidence-Based Clinical Practice Guidelines. Chest. 2012;141(2 Suppl):e531S-75S. Available at: http://www. ncbi.nlm.nih.gov/pmc/articles/PMC3278056/pdf/112304.pdf. Accessed November 17, 2017.

13. Risk factors for stroke and efficacy of antithrombotic therapy in atrial fibrillation. Analysis of pooled data from five randomized controlled trials. Arch Intern Med. 1994;154(13):1449-57.

14. Buckingham TA, Hatala R. Anticoagulants for atrial fibrillation: why is the treatment rate so low? Clin Cardiol. 2002;25(10):447-54.

15. Matchar DB, Samsa GP, Cohen SJ, Oddone EZ, Jurgelski AE. Improving the quality of anticoagulation of patients with atrial fibrillation in managed care organizations: results of the managing anticoagulation services trial. Am J Med. 2002;113(1):42-51.

16. Gallagher A, Rietbrock S, Plumb J, Van Staa T. Initiation and persistence of warfarin or aspirin in patients with chronic atrial fibrillation in general practice: do the appropriate patients receive stroke prophylaxis? J Thromb Haemost. 2008;6(9):1500-06.

17. Hylek EM, Evans-Molina C, Shea C, Henault LE, Regan S. Major hemorrhage and tolerability of warfarin in the first year of therapy among elderly patients with atrial fibrillation. Circulation. 2007;115(21):2689-96.

18. Fiumara K, Goldhaber SZ. A patient's guide to taking Coumadin/warfarin. Circulation. 2009;119(8):e220-22. Available at: http://circ.ahajournals. org/content/119/8/e220.full.pdf+html. Accessed November 17, 2017.

19. Chambers S. Chadda S, Plumb JM. How much does international normalized ratio monitoring cost during oral anticoagulation with a vitamin $\mathrm{K}$ antagonist? A systematic review. Int J Lab Hematol. 2010;32(4):427-42.

20. U.S. Food and Drug Administration, Center for Drug Evaluation and Research. Approval package for Pradaxa. NDA 22-512. October 19, 2010. Available at: https://www.accessdata.fda.gov/drugsatfda_docs/ nda/2010/022512Orig1s000Approv.pdf. Accessed December 18, 2017.

21. Connolly SJ, Ezekowitz MD, Yusuf S, et al. Dabigatran versus warfarin in patients with atrial fibrillation. N Engl J Med. 2009;361(12):1139-51. Available at: http://www.nejm.org/doi/full/10.1056/NEJMoa0905561. Accessed November 17, 2017.

22. Zalesak M, Siu K, Francis K, et al. Higher persistence in newly diagnosed nonvalvular atrial fibrillation patients treated with dabigatran versus warfarin. Circ Cardiovasc Qual Outcomes. 2013;6(5):567-74. Available at: http://circoutcomes.ahajournals.org/content/6/5/567.full.pdf+html. Accessed November 17, 2017.

23. Fonseca E, Sander SD, Hess GP, Ghosh S. Hospital admissions, costs, and 30-day readmissions among newly diagnosed nonvalvular atrial fibrillation patients treated with dabigatran etexilate or warfarin. J Manag Care Spec Pharm. 2015;21(11):1039-53. Available at: http://www.jmcp.org/ doi/10.18553/jmcp.2015.21.11.1039.
24. Bancroft T, Lim J, Wang C, Sander SD, Swindle JP. Health care resource utilization, costs, and persistence in patients newly diagnosed as having nonvalvular atrial fibrillation and newly treated with dabigatran versus warfarin in the United States. Clin Ther. 2016;38(3):545-56.el-6.

25. Sussman M, Ghate S, Sutherland SP, Wang C, Sander S, Griffiths RI. Resource use among nonvalvular atrial fibrillation patients. Am J Pharm Benefits. 2016;8(5):84-92. Available at: http://www.ajpb.com/journals/ ajpb/2016/ajpb_septoct2016/resource-use-among-nonvalvular-atrial-fibrillation-patients. Accessed November 17, 2017.

26. Ezekowitz MD, Connolly S, Parekh A, et al. Rationale and design of RE-LY: randomized evaluation of long-term anticoagulant therapy, warfarin, compared with dabigatran. Am Heart J. 2009;157(5):805-10.

27. Elixhauser A, Steiner C, Harris DR, Coffey RM. Comorbidity measures for use with administrative data. Med Care. 1998;36(1):8-27.

28. Baser O, Palmer L, Stephenson J. The estimation power of alternative comorbidity indices. Value Health. 2008;11(5):946-55.

29. Gage BF, Waterman AD, Shannon W, Boechler M, Rich MW, Radford MJ. Validation of clinical classification schemes for predicting stroke: results from the National Registry of Atrial Fibrillation. JAMA. 2001;285(22):2864-70.

30. Lip GY, Nieuwlaat R, Pisters R, Lane DA, Crijns HJ. Refining clinical risk stratification for predicting stroke and thromboembolism in atrial fibrillation using anovelrisk factor-based approach: the Euro Heart Survey on Atrial Fibrillation. Chest. 2010;137(2):263-72.

31. Gage BF, Yan Y, Milligan PE, et al. Clinical classification schemes for predicting hemorrhage: results from the National Registry of Atrial Fibrillation (NRAF). Am Heart J. 2006;151(3):713-19.

32. Tirschwell DL, Longstreth WT. Validating administrative data in stroke research. Stroke. 2002;33(10):2465-70. Available at: http://stroke.ahajournals. org/content/33/10/2465.full.pdf+html. Accessed November 17, 2017.

33. Boulanger L, Hauch O, Friedman M, et al. Warfarin exposure and the risk of thromboembolic and major bleeding events among Medicaid patients with atrial fibrillation. Ann Pharmacother. 2006;40(6):1024-29.

34. Charlson ME, Pompei P, Ales KL, MacKenzie CR. A new method of classifying prognostic comorbidity in longitudinal studies: development and validation. J Chron Dis. 1987;40(5):373-83.

35. Bellamy L, Rosencher N, Eriksson BI. Adherence to a new oral anticoagulant treatment prescription: dabigatran etexilate. Patient Prefer Adherence. 2009;3:173-77. Available at: http://www.ncbi.nlm.nih.gov/pmc/articles/ PMC2778432/pdf/ppa-3-173.pdf. Accessed November 17, 2017.

36. Wang G, Zhang Z, Ayala C, Dunet DO, Fang J, George MG. Costs of hospitalization for stroke patients aged 18-64 years in the United States. J Stroke Cerebrovasc Dis. 2014;23(5):861-68. 
Health Care Resource Utilization and Costs Among Newly Diagnosed and Oral Anticoagulant Naive Nonvalvular Atrial Fibrillation Patients Treated with Dabigatran or Warfarin in the United States

\begin{tabular}{|c|c|c|c|}
\hline APPE & $\begin{array}{l}\text { IX A Valvu } \\
\text { and } P\end{array}$ & $\begin{array}{l}\text { Heart Dise } \\
\text { cedure Coc }\end{array}$ & Diagnosis \\
\hline Condition & $\begin{array}{l}\text { ICD-9-CM } \\
\text { Diagnosis }\end{array}$ & $\begin{array}{l}\text { ICD-9-CM } \\
\text { Procedure }\end{array}$ & СРТ/HCPCS \\
\hline $\begin{array}{l}\text { Valvular } \\
\text { disease }\end{array}$ & $\begin{array}{l}\text { 394.0x; 394.2x; } \\
\text { 396.0x, 396.1x }\end{array}$ & $\begin{array}{l}35.20,35.22 \\
35.24,35.26 \\
35.28\end{array}$ & \\
\hline $\begin{array}{l}\text { Valvular } \\
\text { procedures }\end{array}$ & & & $\begin{array}{l}\text { 33999, 0257T, } \\
0258 \mathrm{~T}, 0259 \mathrm{~T}, \\
33405,33425, \\
33426,33427, \\
33430,0262 \mathrm{~T}, \\
33475,33460, \\
33463,33464, \\
33465\end{array}$ \\
\hline $\begin{array}{l}\text { CPT }=\text { Currer } \\
\text { Coding Syste } \\
\text { Revision, Cli }\end{array}$ & $\begin{array}{l}\text { cedural Terminolo } \\
\text { D-9-CM = Interna } \\
\text { Modification. }\end{array}$ & $\begin{array}{l}\text { HCPCS = Health } \\
\text { al Classification }\end{array}$ & $\begin{array}{l}\text { Common Procedur } \\
\text { seases, Ninth }\end{array}$ \\
\hline
\end{tabular}


Health Care Resource Utilization and Costs Among Newly Diagnosed and Oral Anticoagulant Naive Nonvalvular Atrial Fibrillation Patients Treated with Dabigatran or Warfarin in the United States

\section{APPENDIX B ICD-9-CM Diagnosis Codes for Bleeding}

\begin{tabular}{|c|c|c|}
\hline Medical Condition & ICD-9-CM Code & Description \\
\hline \multirow{7}{*}{$\begin{array}{l}\text { Intracranial } \\
\text { hemorrhage } \\
\text { (regardless of } \\
\text { position) }\end{array}$} & 430 & Subarachnoid hemorrhage \\
\hline & 431 & Intracerebral hemorrhage \\
\hline & 432 & Other and unspecified intracranial hemorrhage \\
\hline & $852.0 \mathrm{x}$ & Subarachnoid hemorrhage following injury without mention of open intracranial wound \\
\hline & $852.2 \mathrm{x}$ & Subdural hemorrhage following injury without mention of open intracranial wound \\
\hline & $852.4 \mathrm{x}$ & Extradural hemorrhage following injury without mention of open intracranial wound \\
\hline & 853.0 & Other and unspecified intracranial hemorrhage following injury without mention of open intracranial wound \\
\hline \multirow{40}{*}{$\begin{array}{l}\text { Gastrointestinal } \\
\text { (primary) }\end{array}$} & 455.2 & Internal hemorrhoids with other complication \\
\hline & 455.5 & External hemorrhoids with other complication \\
\hline & 455.8 & Unspecified hemorrhoids with other complication \\
\hline & 456.0 & Esophageal varices with bleeding \\
\hline & 456.20 & Esophageal varices in disease classified elsewhere, with bleeding \\
\hline & 459.0 & Hemorrhage, unspecified \\
\hline & 530.7 & Gastroesophageal laceration-hemorrhage syndrome \\
\hline & 530.82 & Esophageal hemorrhage \\
\hline & 531.0 & Acute gastric ulcer with hemorrhage \\
\hline & 531.2 & Acute gastric ulcer with hemorrhage and perforation \\
\hline & 531.4 & Chronic or unspecified gastric ulcer with hemorrhage \\
\hline & 531.6 & Chronic or unspecified gastric ulcer with hemorrhage and perforation \\
\hline & 532.0 & Acute duodenal ulcer with hemorrhage \\
\hline & 532.2 & Acute duodenal ulcer with hemorrhage and perforation \\
\hline & 532.4 & Chronic or unspecified duodenal ulcer with hemorrhage \\
\hline & 532.6 & Chronic or unspecified duodenal ulcer with hemorrhage and perforation \\
\hline & 533.0 & Acute peptic ulcer of unspecified site with hemorrhage \\
\hline & 533.2 & Acute peptic ulcer of unspecified site with hemorrhage and perforation \\
\hline & 533.4 & Chronic or unspecified peptic ulcer of unspecified site with hemorrhage \\
\hline & 533.6 & Chronic or unspecified peptic ulcer of unspecified site with hemorrhage and perforation \\
\hline & 534.0 & Acute gastrojejunal ulcer with hemorrhage \\
\hline & 534.2 & Acute gastrojejunal ulcer with hemorrhage and perforation \\
\hline & 534.4 & Chronic or unspecified gastrojejunal ulcer with hemorrhage \\
\hline & 534.6 & Chronic or unspecified gastrojejunal ulcer with hemorrhage and perforation \\
\hline & 535.01 & Acute gastritis, with hemorrhage \\
\hline & 535.11 & Atrophic gastritis, with hemorrhage \\
\hline & 535.21 & Gastric mucosal hypertrophy, with hemorrhage \\
\hline & 535.31 & Alcoholic gastritis, with hemorrhage \\
\hline & 535.41 & Other specified gastritis, with hemorrhage \\
\hline & 535.51 & Unspecified gastritis and gastroduodenitis, with hemorrhage \\
\hline & 535.61 & Duodenitis, with hemorrhage \\
\hline & 537.83 & Angiodysplasia of stomach and duodenum with hemorrhage \\
\hline & 562.02 & Diverticulosis of small intestine with hemorrhage \\
\hline & 562.03 & Diverticulitis of small intestine with hemorrhage \\
\hline & 562.12 & Diverticulosis of colon with hemorrhage \\
\hline & 562.13 & Diverticulitis of colon with hemorrhage \\
\hline & 568.81 & Hemoperitoneum (nontraumatic) \\
\hline & 569.3 & Hemorrhage of rectum and anus \\
\hline & 569.85 & Angiodysplasia of intestine with hemorrhage \\
\hline & 578 & Gastrointestinal hemorrhage \\
\hline \multirow{7}{*}{$\begin{array}{l}\text { Other bleeding } \\
\text { (primary) }\end{array}$} & 599.7 & Hematuria \\
\hline & $719.1 \mathrm{x}$ & Hemarthrosis \\
\hline & 786.3 & Hemoptysis \\
\hline & 423.0 & Hemopericardium \\
\hline & 593.81 & Vascular disorders of kidney \\
\hline & 784.7 & Epistaxis \\
\hline & 784.8 & Hemorrhage from throat \\
\hline
\end{tabular}

ICD-9-CM = International Classification of Diseases, Ninth Revision, Clinical Modification. 